\title{
Fundamental Study on a Thin-Film AE Sensor for Measurement of Behavior of a Multi-Pad Contact Slider
}

\author{
Satomitsu Imai \\ Data Storage \& Retrieval Systems Division, Hitachi, Ltd., 2880 Kozu, Odawara, Kanagawa, 256 Japan
}

Gert-Jan-Burger, T. S. J. Lammerink, J. H. J. Fluitman

MESA Research Institute, University of Twente, 7500 AE Enschede, The Netherlands

\begin{abstract}
To study the fundamental dynamic characteristics of a multi-pad slider for contact recording, we developed a thinfilm piezoelectric acoustic emission array sensor on an Sisuspension with an array pattern similar to that of contact pads. Experiments showed that the sensitivity of the sensor is about 0 . $11 \mathrm{~V} / \mathrm{N}$ (slider thickness: $0.2 \mathrm{~mm}$ ) and that each array sensor designed here is not influenced by the acoustic waves which occur due to contact with other contact pads, so the contact or non-contact condition of each pad can be measured.
\end{abstract}

\section{I NTRODUCTION}

We developed a thin-film piezoelectric $\mathrm{AE}$ sensor to measure the behavior of contact sliders (about $25 \%$ size). This type of slider usually has contact pads; the condition of each pad can be determined by using an arrayed thin-film AE sensor. A slider can be used by gluing it on the array sensor, so that a real slider can be tested. In this sensor system, the suspension material is $\mathrm{Si}$. The suspension was designed to satisfy the required low stiffness for contact recording.

\section{PURPOSE OF THE SENSOR IN MEASUREMENT}

Fig. 1 shows what we wish to measure for the sensor we developed. A contact slider usually has several contact pads. It is important to know which contact pad is in contact and which one is not in contact with a disk surface to assess the wear of the contact pads and the spacing between the head and disk surface. Since the head is fabricated in one of the pads, if we get an output of the sensor element corresponding to the pad, it means that the head is in contact with the disk surface. We aimed the sensor we developed as an experimental tool for mainly a non-flying type contact slider. We chose a thin-film piezoelectric AE sensor to realize the array sensor and small sensor[1].

\section{S TRUCTURE OF THE SENSOR SYSTEM}

Fig. 2 shows the suspension and array sensor system. Micromachine technique was used in the fabrication. The thin-film AE array sensors were fabricated on $\mathrm{Si}$-suspensions. We made 3 array patterns of the thin-film sensor. Fig. 3 shows the structure of the thin-film $\mathrm{AE}$ sensor. A slider with contact pads is glued on the array sensor. The acoustic emission wave generated in the contact pad propagates to the Si-suspension backing material through the thin-film AE sensor. If the slider is thin, we can get high sensitivity. The position of each contact pad and each sensor element must be matched when gluing the slider.

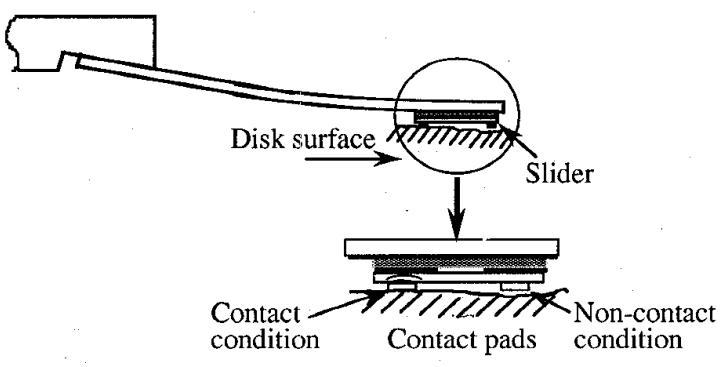

Fig. 1. Array sensor to detect contact or non-contact condition of pads on a slider

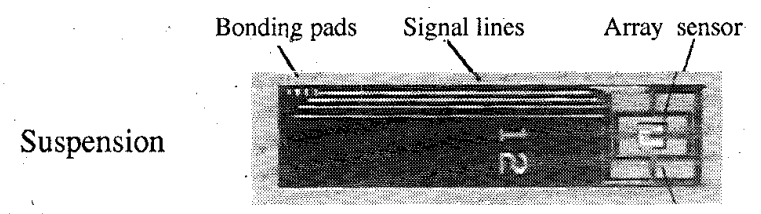

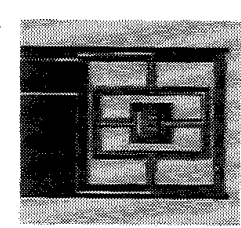

1-sensor type

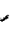

Fig. 2. Si suspension with the thin-film AE array sensor

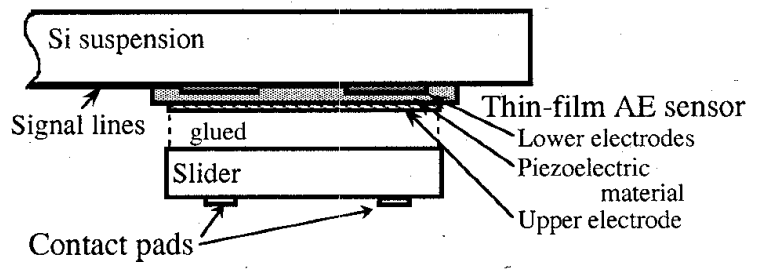

Fig. 3. Structure of the array sensor and the suspension

\section{DESIGN OF THE ARRAY SENSOR}

Each sensor element is composed of the sputtered $\mathrm{ZnO}$ piezoelectric material (thickness: $0.002 \mathrm{~mm}$ ) and the lower arrayed and upper electrodes as shown in Fig. 3. Fig. 4 shows the sizes and array patterns of the sensor. We chose 3 array 
patterns of they sensor to examine the relation between the number of pads and the dynamic characteristics of the slider. A multi-pad slider has the advantage of low wear. In one pad slider, there is a possibility that a certain part of the pad is in contact with the disk surface. It may lead to large spacing in the head position. In case of the 2-pad slider like 2 sensor type in Fig. 4, the pitching moment acts in the slider by the loading force and it makes the slider follow the disk surface in the slider pitching direction. Similarly, in case of 3-pad slider like 3 sensor type in Fig. 4, the pitching and rolling moments act in the slider by the loading force and they make the slider follow the disk surface in the slider pitching and rolling directions.

The sensor dimensions were determined by the following sensor output simulations. The sensor output was calculated by (1) [1] and the stress in the piezoelectric layer was calculated by (2). The input force is assumed to be a half sine wave here (period: $2 \mu \mathrm{s}$, maximum amplitude: $1 \mathrm{~N}$ ).

Fig. 5 shows the relation between sensor output and the distance between the sensor and the contact force. An acoustic emission wave due to contact propagates in all directions from the pad. Therefore, it is important in designing array sensor that sensor output is not influenced by the contact forces in other contact pads. When the distance $r$ in Fig. 5 is larger than $0.6 \mathrm{~mm}$ for the sensors larger than 0.3 $\mathrm{mm} \times 0.3 \mathrm{~mm}$, the sensor output is almost zero. Therefore, we chose $0.6 \mathrm{~mm}$ as the distance and $0.3 \mathrm{~mm} \times 0.3 \mathrm{~mm}$ as the sensor area.

Fig. 6 shows the relation between the thickness of the slider and the maximum output of the sensor. A thinner slider increases the sensitivity of the sensor. The slider thickness less than $0.2 \mathrm{~mm}$ is good to get high sensitivity.

$$
U=\sigma_{z} t_{p} h_{33} s_{33}^{D} \frac{C_{p}}{C_{p}+C_{e}}
$$

$U:$ Sensor output voltage (V)

$\sigma_{z}:$ Stress of the piezoelectric layer $\left(\mathrm{N} / \mathrm{m}^{2}\right)$

$t_{p}$ : Thickness of the piezoelectric layer $(0.002 \mathrm{~mm})$

$h_{33}$ : Piezoelectric constant ( $\mathrm{ZnO}:-3.29 \times 10^{10} \mathrm{~N} / \mathrm{C}$ )

$S_{33}$ : Compliance ( $\left.\mathrm{ZnO}: 5.13 \times 10^{-12} \mathrm{~m}^{2} / \mathrm{N}\right)$

$C_{p}$ : Capacitance of the sensor element

(In case of the sensor size $0.3 \times 0.3, \mathrm{Cp}=3.28 \mathrm{pF}$ )

$C_{e}$ : Parasitic capacitance of the measuring system

(63 $\mathrm{pF}$ by the experiment in Fig. 9)

$$
\begin{aligned}
\sigma_{\mathrm{z}}= & 1 / A \int_{\mathrm{A}} \sigma_{\mathrm{z} 0} \mathrm{~d} A . \\
& \sigma_{\mathrm{z} 0}=-(3 \mathrm{~F} / 2 \pi) \mathrm{z}^{3}\left(\mathrm{z}^{2}+\mathrm{r}^{2}\right)-5 / 2
\end{aligned}
$$

$z$ : Thickness of the slider

$r$ : Horizontal distance between the contact force and the center of the sensor element

A: Area of the sensor element

\section{DESIGN OF THE SUSPENSION}

Fig. 7 shows the shape and sizes of the sensor-suspension. Suspension material is $\mathrm{Si}$. The stiffness of the suspension and gimbal was designed to be suitable for contact recording. The suspension spring part and the gimbal beams are half-etched
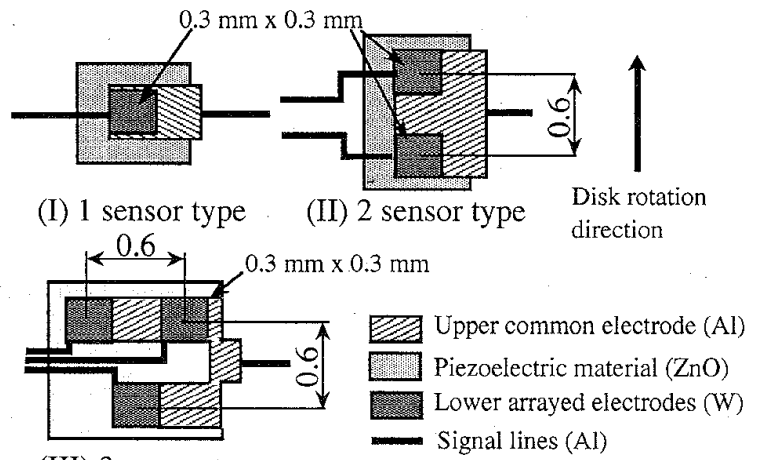

(III) 3 sensor type

Fig. 4. Sizes and patterns of the array sensor

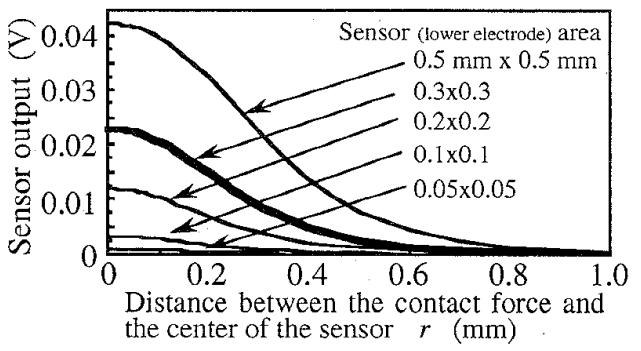

Fig. 5. Simulated relation between sensor output and the distance between the sensor and the contact force (Stress was calculated by (2), where $z=0.38 \mathrm{~mm}$.)

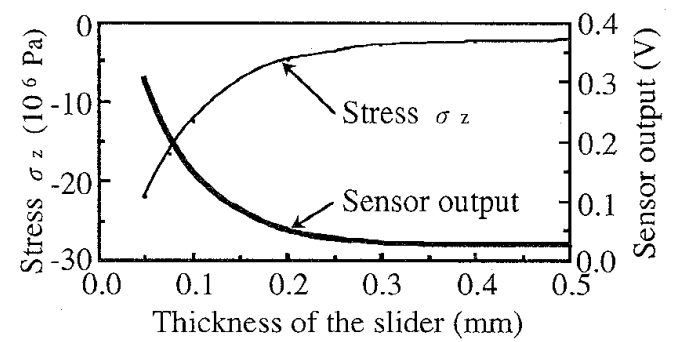

Fig. 6. Simulated relation between the thickness of the slider and the maximum output of the sensor (Slider material: $\mathrm{Al}_{2} \mathrm{O}_{3} \cdot \mathrm{TiC}$ )

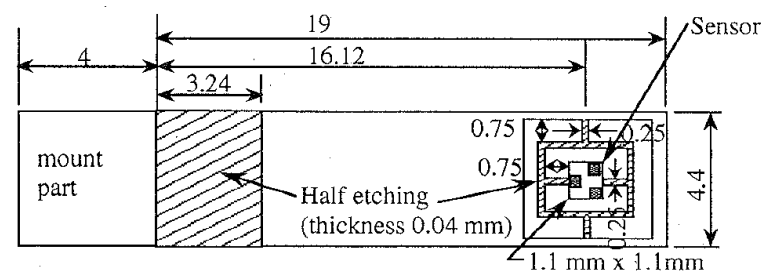

Fig. 7. Shape and sizes of the suspension

to a thickness of $0.04 \mathrm{~mm}$ for low stiffness .

Table I shows the calculated stiffness and natural frequencies of the fabricated suspension (based on the finite element method). A loading force of the slider was designed as $1.5 \mathrm{mN}$.

The suspension and gimbal dimensions were determined by the following reasons. The stiffness in the loading direction is less than $10 \mathrm{mN} / \mathrm{mm}$ to make change of the loading force due 
to disk run-out (we assumed about $15 \mu \mathrm{m}$ ) less than $10 \%$ of the designed value. The suspension was designed to allow change of slider pitching and rolling angles in some degree for suspension mounting error and disk run-out. From the equilibrium of the pitching and rolling stiffness of the gimbal-suspension and the pitching and rolling moments in the slider by the reaction forces in the pads due to the loading force, the allowed angle change of the slider in the pitching and rolling directions are about 0.11 and 0.09 degree.

Fig. 8 shows the fabrication process. We chose the following fabrication steps not to damage the sensor when half-etching the suspension and gimbal. In step I, the suspension spring part and gimbal is half-etched on one side of the suspension. Then, the sensor array is fabricated on the other surface of the suspension as shown in step II -IV. In step $\mathrm{V}$, the gimbal beams are dry etched on $\mathrm{SF}_{6}: \mathrm{O}_{2}$ with a chromium mask to protect the sensor array and signal lines from the etching gases. The gimbal beams are etched to the specified width.

\section{CHARACTERISTICS}

An experiment on the basic characteristics of the sensor output was conducted to check the sensor sensitivity and whether the sensor output is not influenced by the contact force on other pads.

The test was conducted by dropping a ball on one of the sensor elements (3-sensor type in Fig. 4) and we examined the outputs of the two sensors (sensor A and B in Fig 9). In this test, the specification of the slider is shown in Fig. 9.

The maximum outputs of the two sensors are plotted. From the maximum output of the sensor $A$ on which the ball was dropped, the sensitivity of the sensor is about $0.11 \mathrm{~V} / \mathrm{N}$. The output of the sensor B which is $0.6 \mathrm{~mm}$ away from the sensor $A$ is almost zero. Therefore, we confirmed that the output of this array sensor is hardly influenced by the contact force on other pads.

We checked the result of the experiment by the simulation. The calculated stress distribution of the piezoelectric layer is shown in Fig. 9. The stress in the sensor B is almost zero and the simulated output of the sensor B is also almost zero. This result is the same as that of the experiment. We confirmed that the arrayed sensor is not influenced by the contact force on other pads also in the simulation.

\section{S UMMARY}

We developed a thin-film $\mathrm{AE}$ array sensor to measure the contact or non-contact condition of contact pads on the slider, and confirmed its validity by simulation and experiment. The sensor output sensitivity is about $0.11 \mathrm{~V} / \mathrm{N}$ for a slider thickness of $0.2 \mathrm{~mm}$.

\section{R EFERENCES}

[1] S. Imai, "A thin-film piezoelectric impact sensor array fabricated on a Si slider for measuring head-disk interaction," IEEE Transactions on Magnetics, Vol. 31, No. 6, pp. 3009-30 $11,1995$.

[2] S. P. Timoshenko and J. N. Gooddier, Theory of Elasticity, McGraw-Hill Books, (1934), 413 - 417.
Table I Stiffness and natural frequencies

\begin{tabular}{lr}
\hline Loading force $\mathrm{mN}$ & 1.5 \\
\hline Suspension and gimbal stiffness & \\
Loading-dir. mN/mm & 9.12 \\
Pitch $\mathrm{mNmm} / \mathrm{rad}$. & 457 \\
Roll mNmm/rad. & 604 \\
\hline Natural frequencies $\mathrm{kHz}$ & \\
Bending & 1.57 \\
Torsion & 2.30 \\
Sway & 15.52 \\
\hline
\end{tabular}

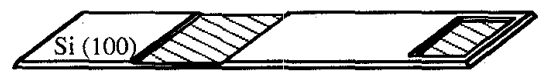

(I) Wet etching (KOH) of the suspension spring part and gimbal (thickness: $0.2 \mathrm{~mm} \rightarrow 0.04 \mathrm{~mm}$ )

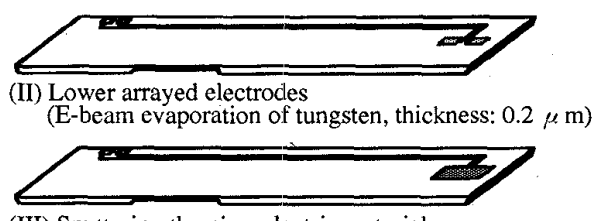

(III) Sputtering the piezoelectric material ( $\mathrm{ZnO}, \mathrm{RF}$ sputtering of zinc target in an oxygen environment)

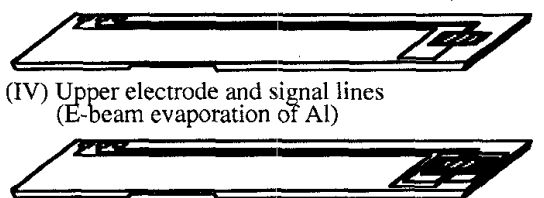

(V) Fabrication of the gimbal beams

(Anisotropic etching of $\mathrm{Si}$ by the reactive ion etching of SF 6: $\mathrm{O}_{2}$ $=28: 5 \mathrm{sccm}$ with a chromum mask)

Fig. 8. Fabrication of the sensor and suspension
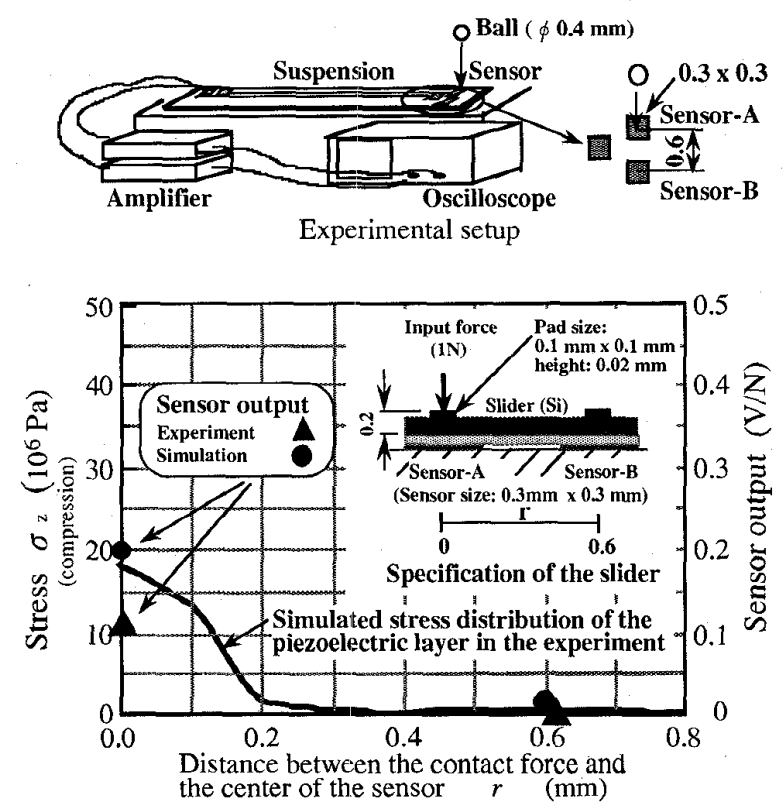

Fig. 9. Sensor output characteristics (In sensor output simulation, the capacitance of each sensor element is $3.28 \mathrm{pF}$ and the parasitic capacitance is about $63 \mathrm{pF}$.) 